\title{
COMPLEX RETRACTIONS AND ENVELOPES OF HOLOMORPHY
}

\author{
S. TRAPANI
}

(Communicated by Irwin Kra)

\begin{abstract}
In this paper we show that if a domain $\Omega$ of a Stein manifold $X$ is a "holomorphic deformation retract" of a domain of holomorphy $D \subseteq X$, then $\Omega$ has a univalent envelope of holomorphy $\Omega^{*} \subseteq X$.
\end{abstract}

1. Introduction. Let $X$ be a connected Stein manifold of dimension $n>1$. Let $D$ and $\Omega$ be domains in $X$ with $\Omega \subseteq D$. In this paper we study the pairs $(D ; \Omega)$ such that $D$ is a domain of holomorphy which can be deformed inside $\Omega$ with a continuous family of holomorphic maps (see Definition 1). It will be shown that the domain $\Omega$ has a univalent envelope of holomorphy $\Omega^{*} \subseteq X$. Under some additional hypotheses, we shall prove that $\left(D ; \Omega^{*}\right)$ is a Runge pair.

2.

DEFINITION 1. Let $\Omega \subseteq D$ be domains of a Stein manifold $X$; we say that the continuous map $F:[0 ; 1] \times D \rightarrow X$ is a complex retraction of $D$ in $\Omega$ if the following conditions are fulfilled:

(1) $F(0)=$ Id,

(2) $F(1 ; D) \subseteq \Omega$,

(3) $F(t): D \rightarrow X$ is holomorphic, $\forall t \in[0 ; 1]$,

(4) $F(t ; \Omega) \subseteq \Omega, \forall t \in[0 ; 1]$.

THEOREM 1. Let $D \supseteq \Omega$ be a pair of domains in $X$ with $D$ being a domain of holomorphy; let $F:[0 ; 1] \times D \rightarrow X$ be a complex retraction of $D$ in $\Omega$. Then $\Omega$ has a univalent envelope of holomorphy $\Omega^{*} \subseteq D \subseteq X$ and $F\left(t ; \Omega^{*}\right) \subseteq \Omega^{*}, \forall t \in[0 ; 1]$.

LEMMA 1. Let $V$ and $W$ be Stein manifolds, let $\Delta_{1} \subseteq V$ and $\Delta_{2} \subseteq W$ be domains, let $\left(\tilde{\Delta}_{1} ; \pi_{1}\right)$ and $\left(\tilde{\Delta}_{2} ; \pi_{2}\right)$ their Riemann domains envelopes of holomorphy, let $i_{1}: \Delta_{1} \rightarrow \tilde{\Delta}_{1}, i_{2}: \Delta_{2} \rightarrow \tilde{\Delta}_{2}$ be the canonical embeddings.

If $f: V \rightarrow W$ is a holomorphic map such that $f\left(\Delta_{1}\right) \subseteq \Delta_{2}$, then there exists a unique map $\tilde{f}: \tilde{\Delta}_{1} \rightarrow \tilde{\Delta}_{2}$ such that the diagram

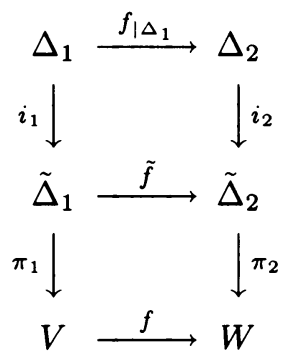

Received by the editors September 3, 1986 and, in revised form, July 23, 1987.

1980 Mathematics Subject Classification (1985 Revision). Primary 32D10. 
is commutative. So, $f\left(\pi_{1}\left(\tilde{\Delta}_{1}\right)\right) \subseteq \pi_{2}\left(\tilde{\Delta}_{2}\right)$. Moreover, if $F:[0 ; 1] \times V \rightarrow W$ is continuous, $F(t)$ is holomorphic $\forall t \in[0 ; 1]$ and $F(t)\left(\Delta_{1}\right) \subseteq \Delta_{2} \forall t \in[0 ; 1]$, then $\tilde{F}:[0 ; 1] \times \tilde{\Delta}_{1} \rightarrow \tilde{\Delta}_{2}$ is continuous.

PROOF. Let us take the map $\tilde{f}=i_{2} \circ f \circ \pi_{1}: i_{1}\left(\Delta_{1}\right) \rightarrow \tilde{\Delta}_{2}$. Now regard $\tilde{\Delta}_{2}$ as a closed submanifold of $\mathbf{C}^{N}(N=2 n+1)$ and consider $\tilde{f}: i_{1}\left(\Delta_{1}\right) \rightarrow \mathbf{C}^{N}$. This map can be extended to $\tilde{f}: \tilde{\Delta}_{1} \rightarrow \mathbf{C}^{N}$. We must prove that $\tilde{f}\left(\tilde{\Delta}_{1}\right) \subseteq \tilde{\Delta}_{2}$. Choose a point $p \in \mathbf{C}^{N}-\tilde{\Delta}_{2}$ and an open set of holomorphy $A \subseteq \mathbf{C}^{N}$ such that $p \notin A \supseteq \tilde{\Delta}_{2}$; we know that $\tilde{f}^{-1}(A)$ is a Stein open set in $\tilde{\Delta}_{1}$ containing $i_{1}\left(\Delta_{1}\right)$, so $\tilde{f}^{-1}(A)=\tilde{\Delta}_{1}$ and $p \notin \tilde{f}\left(\tilde{\Delta}_{1}\right)$.

Commutativity of the diagram and uniqueness of $\tilde{f}$ follow from uniqueness of analytic continuation. Now let $F:[0 ; 1] \times V \rightarrow W$ be as above, and take $t_{n} \rightarrow t_{0}$ in $[0 ; 1]$. It follows from Vitali's theorem that $F\left(t_{n}\right) \rightarrow F\left(t_{0}\right)$ uniformly on the compact sets of $V$, and thus $\tilde{F}\left(t_{n}\right) \rightarrow \tilde{F}\left(t_{0}\right)$ on the compact sets of $i_{1}\left(\Delta_{1}\right)$.

Let $\left\{K_{n}\right\}_{n \in N}$ be an exhaustion of $i_{1}\left(\Delta_{1}\right)$ by compact sets, then $\bigcup_{n \in N}\left(\hat{K}_{n}\right)_{\tilde{\Delta}_{1}}$ is an open subset of holomorphy of $\tilde{\Delta}_{1}$. It follows that $\tilde{F}\left(t_{n}\right) \rightarrow \tilde{F}\left(t_{0}\right)$ on the compact sets of $\tilde{\Delta}_{1}$ and, thus, $\tilde{F}:[0 ; 1] \times \tilde{\Delta}_{1} \rightarrow \tilde{\Delta}_{2}$ is continuous.

PROOF OF THEOREM 1 . Let $(\tilde{\Omega} ; \pi)$ be the envelope of holomorphy of $\Omega$; we have $\Omega^{*}=\pi(\tilde{\Omega}) \subseteq D$. Choose a point $0 \in \Omega$. A curve $\gamma$ in $X$ joining 0 and a point $P \in \Omega^{*}$ is such that there exists a curve $\tilde{\gamma}$ starting from $0 \in \tilde{\Omega}$ with $\pi(\tilde{\gamma})=$ $\gamma$ if and only if every holomorphic function $f: \Omega \rightarrow \mathbf{C}$ can be holomorphically continued along $\gamma[\mathbf{4}]$. We will call this kind of curve a liftable curve. Let us consider the continuous map $\tilde{F}:[0 ; 1] \times \tilde{\Omega} \rightarrow \tilde{\Omega}$ defined by Lemma 1 , where $V=D$ and $W=X$. Take a liftable curve $\gamma=\pi(\tilde{\gamma})$ joining $0 \in \Omega$ and $P \in \pi(\tilde{\Omega})$. Then $F(t ; \gamma)=F(t ; \pi(\tilde{\gamma}))=\pi \tilde{F}(t ; \tilde{\gamma})$ is a liftable curve joining $F(t ; 0)$ and $F(t ; p)$. The curve $C_{p}(t)=F(1-t ; \gamma(1))=F(1-t ; \pi \tilde{\gamma}(1))=\pi(\tilde{F}(1-t ; \gamma(1)))$ is also liftable. Moreover, the curve $C_{0}(t)=F(t ; 0)$ has support in $\Omega$. We now define the curve $\phi(t ; \gamma)$ in the following way:

$$
\begin{aligned}
\phi(t ; \gamma)(s)=C_{0}(3 s t) & \text { if } 0 \leq s \leq \frac{1}{3} \\
\phi(t ; \gamma)(s)=F(t ; \gamma(3 s-1)) & \text { if } \frac{1}{3} \leq s \leq \frac{2}{3} \\
\phi(t ; \gamma)(s)=C_{p}(3 t(s-1)+1) & \text { if } \frac{2}{3} \leq s \leq 1
\end{aligned}
$$

Every curve $\phi(t ; \gamma)$ is a liftable curve joining 0 and $P$. The map $\phi(t ; s)=\phi(t ; \gamma)(s)$ is a homotopy between $\gamma$ and $\phi(1 ; \gamma)$. Now, if we take a function $f$ holomorphic in $\Omega$ and extend this function along $\phi(1 ; \gamma)$, we see that the value in $P$ of the extension does not depend on $\gamma$, but only on $C_{p}$. Hence the monodromy theorem [4] implies that the value in $P$ of the extension of $f$ along $\gamma$ does not depend on $\gamma$. This is our thesis. The fact that $F\left(t ; \Omega^{*}\right) \subseteq \Omega^{*} \forall t \in[0 ; 1]$ follows by Lemma 1 .

THEOREM 2. Let $\Delta \subseteq \Omega$ be domains of $X$ with $\Delta$ a domain of holomorphy, and assume that there exists a continuous map $F:[0 ; 1] \times \Omega \rightarrow \Omega$ which fulfills conditions 1,2 and 3 of Definition 1 with respect to the pair $(\Omega ; \Delta)$. Under these assumptions, the following facts are equivalent:

(a) The domain $\Omega$ has a univalent envelope of holomorphy $\Omega^{*} \subseteq X$. 
(b) There exists a domain of holomorphy $D \supseteq \Omega$ and a family $\tilde{F}(t)$, of holomorphic maps from $D$ to $X, t \in[0 ; 1]$ such that $\tilde{F}(t)$ extends $F(t)$ for every $t \in[0 ; 1]$.

(c) There exists a domain of holomorphy $D \supseteq \Omega$ and a complex retraction of $D$ in $\Omega$.

ProOF. (a) $\Rightarrow$ (b) follows by Lemma 1 .

(c) $\Rightarrow($ a) by Theorem 1 .

(b) $\Rightarrow(\mathrm{c})$. Consider the set $L$ equal to the intersection of all the domains of holomorphy containing $\Omega$; then, since $\Omega$ is open and connected, the interior of $L$, which we shall denote by $\tilde{D}$, is a domain of holomorphy contained in $D[7]$. The maps $\tilde{F}(t): \tilde{D} \rightarrow X$ define a map $\tilde{F}:[0 ; 1] \times \tilde{D} \rightarrow X$. We wish to prove that $\tilde{F}$ is a complex retraction of $\tilde{D}$ in $\Omega$. We then have to check conditions 1 to 4 of Definition 1. Condition 1 follows from the uniqueness of analytic continuation. We know that $(\tilde{F}(1))^{-1}(\Delta)$ is an open set of holomorphy containing $\Omega$, then $\tilde{D} \subseteq(\tilde{F}(1))^{-1}(\Delta)$ and $\tilde{F}(1 ; \tilde{D}) \subseteq \Delta \subseteq \Omega$. Therefore, condition 2 is verified.

Conditions (3) and (4) follow by the definition of $\tilde{F}$. Continuity of $\tilde{F}$ follows as shown in Lemma 1.

THEOREM 3. Let $\Omega \subseteq D$ be domains of $X$; suppose that $D$ is a domain of holomorphy; let $F:[0 ; 1] \times D \rightarrow X$ be a complex retraction of $D$ in $\Omega$ and assume that $F$ fulfills the following additional conditions:

(i) $F(t ; D) \subseteq D \forall t \in[0 ; 1]$,

(ii) $F(t)$ is one-to-one $\forall t \in[0 ; 1[$,

(iii) $(D ; F(t ; D))$ is a Runge pair $\forall t \in[0 ; 1[$.

Then, if $\Omega^{*}$ denotes the envelope of $\Omega,\left(D ; \Omega^{*}\right)$ is a Runge pair.

ProOF. Let $K \subseteq \Omega^{*}$ be a compact set; we must prove that $\hat{K}_{D} \subseteq \Omega^{*}$. By Theorem 1 , we have established that $F\left(t ; \Omega^{*}\right) \subseteq \Omega^{*}, \forall t \in[0 ; 1]$. Observe that the set $K_{1}=(F([0 ; 1] \times K)) \widehat{\Omega^{*}}$ is compact in $\Omega^{*}$. Notice now that if we choose $t \in[0 ; 1[$ such that $F\left(t ; \hat{K}_{D}\right) \subseteq \Omega^{*}$, then $F\left(t ; \hat{K}_{D}\right) \subseteq K_{1}$. Indeed, since $F(t): D \rightarrow F(t ; D)$ is a biholomorphic map and $(D ; F(t ; D))$ is a Runge pair, we have

$$
F\left(t ; \hat{K}_{D}\right)=\left(F(t ; K) \hat{F}_{(t ; D)}=F(t ; K) \hat{D}_{D}\right.
$$

Therefore, the inclusion $(F(t ; K))_{D} \subseteq \Omega^{*}$ implies that $(F(t ; K))_{D} \subseteq(F(t ; K)) \widehat{\Omega^{*}} \subseteq$ $K_{1}[\mathbf{5}]$.

Now observe that the set $A=\left\{t \in[0 ; 1]: F\left(t ; \hat{K}_{D}\right) \subseteq \Omega^{*}\right\}$ is open and nonempty in $[0 ; 1]$; in fact, $1 \in A$ and if $t_{0} \in A$, since $\hat{K}_{D}$ is compact and $\Omega^{*}$ is open, we can find an open neighborhood of $t_{0}$ in $[0 ; 1]$ contained in $A$. Hence, $A-\{1\}$ is open and nonempty in $\left[0 ; 1\left[\right.\right.$; moreover $A-\{1\}=\left\{t \in\left[0 ; 1\left[: F\left(t ; \hat{K}_{D}\right) \subseteq K_{1}\right\}\right.\right.$, which is closed in $\left[0 ; 1\left[\right.\right.$. Therefore $A=[0 ; 1]$ and, thus, $F\left(0 ; \hat{K}_{D}\right)=\hat{K}_{D} \subseteq \Omega^{*}$.

EXAMPLES. (1) Let $X=\mathbf{C}^{N}$, let $\Omega$ be an open set containing 0 , and star-shaped with respect to 0 . Then $\Omega$ has a univalent envelope of holomorphy $\Omega^{*} \subseteq \mathbf{C}^{N}$ and $\left(\Omega^{*} ; \mathbf{C}^{N}\right)$ is a Runge pair. This is a consequence of Theorems (1) and (3) considering the following map, $F:[0 ; 1] \times \mathbf{C}^{N} \rightarrow \mathbf{C}^{N}: F(t ; z)=(1-t) z$. A different proof of this fact was given in $[\mathbf{1}]$.

(2) Let $X=\mathbf{C}^{N}$, and let $\Omega$ be a domain satisfying the following properties:

(a) There exists $\left(\alpha_{1} \cdots \alpha_{n}\right) \in \mathbf{N}^{n}$ such that if $z \in \Omega$ and $t \in[0 ; 1],\left(t^{\alpha_{1}} z_{1}\right.$; $\left.t^{\alpha_{2}} z_{2} ; \ldots ; t^{\alpha_{n}} z_{n}\right) \in \Omega$. 
(b) If $\left(i_{1} \cdots i_{k}\right)$ is the ordered set of indices such that $\alpha_{i_{k}}=0$ and $\pi_{i_{1} \cdots i_{k}}: \mathbf{C}^{n} \rightarrow$ $\mathrm{C}^{k}$ denotes the canonical projection, then $\pi_{i_{1} \cdots i_{k}}(\Omega)$ is a domain of holomorphy.

Under these assumptions, the domain $\Omega$ has a univalent envelope of holomorphy $\Omega^{*} \subseteq \mathbf{C}^{n}$ and $\left(\pi_{i_{1} \cdots i_{k}}(\Omega) \times \mathbf{C}^{n-k} ; \Omega^{*}\right)$ is a Runge pair.

This can be shown by considering the map $F:[0 ; 1] \times \pi_{i_{1} \cdots i_{k}}(\Omega) \times \mathbf{C}^{n-k} \rightarrow \mathbf{C}^{n}$ :

$$
F\left(t ; z_{1} ; \ldots ; z_{n}\right)=\left((1-t)^{\alpha_{1}} z_{1} ; \ldots ;(1-t)^{\alpha_{n}} z_{n}\right) .
$$

COUNTEREXAMPLE. Note that if $\Omega \subseteq \mathbf{C}^{n}$ is star-shaped with respect to the origin, then $\Omega$ fulfills the hypotheses of Theorem 2 ; therefore, every domain that is biholomorphic to a star-shaped domain fulfills the same hypotheses. We will now exhibit a domain $\Omega \subseteq \mathbf{C}^{2}$ which is biholomorphic to a star-shaped domain; however, it does not have a univalent envelope of holomorphy in $\mathbf{C}^{2}$. Let $\Omega=$ $\left\{(z ; w) \in \mathbf{C}^{2}:|| z|-1|<\frac{1}{2} ; \exists \theta>0\right.$ such that $z=|z| e^{i \theta}$ and ||$\left.w|-\theta|<\frac{1}{2}\right\}$. It is well known that $\Omega$ has no univalent envelope in $\mathbf{C}^{2}[\mathbf{3}]$. However, the map $F(z ; w)=(\log z ; w)$ is a biholomorphism of $\Omega$ with a star-shaped domain.

ADDENDUM. In a forthcoming paper E. Casadio Tarabusi gives an example of a domain $\Omega \subseteq \mathbf{C}^{2}$ with the following properties:

(a) $\Omega$ has a univalent envelope of holomorphy $\Omega^{*} \subseteq \mathbf{C}^{2}$;

(b) There exists a continuous map $F:[0,1] \times \Omega^{*} \rightarrow \Omega^{*}$ fulfilling conditions (1), (2), (4) of Definition 1 (in fact $\Omega$ is a deformation retract of $\Omega^{*}$ );

(c) There is no complex retraction of $\Omega^{*}$ in $\Omega$.

\section{BIBLIOGRAPHY}

1. B. Almer, Sur quelques problèmes de la théorie..., Arkiv für Mat. Astronomy och Fysik B.d. 17 (7) (1922).

2. S. Bochner and W. T. Martin, Several complex variables, Princeton Univ. Press, Princeton, N. J., 1948, 366.

3. R. Carmignani, Envelope of holomorphy and holomorphic convexity, Trans. Amer. Math. Soc. 179 (1973), 415-431.

4. S. Coen, Una introduzione ai domini di Riemann non ramificati $n$ dimensionali, Pitagora Ed., Bologna, 1980.

5. J. E. Fornaess and W. H. Zame, Riemann domains and envelopes of holomorphy, Duke Math. J. 50 (1983), 273-283.

6. R. Gunning and H. Rossi, Analytic functions of several complex variables, Prentice-Hall, 1965.

7. L. Hörmander, An introduction to complex analysis in several variables, Van Nostrand, 1966.

Dipartimento di Matematica, Universita di Pisa, Via Buonarrots 2, 56100 PISA, ITALY

Current address: Department of Mathematics, Harvard University, Science Center, Cambridge, Massachusetts 02138 